\title{
Stage III Thymic Epithelial Neoplasm AJCC v8
}

National Cancer Institute

\section{Source}

National Cancer Institute. Stage III Thymic Epithelial Neoplasm A/CC V8. NCI Thesaurus. Code C136325.

Stage III includes: IIIA: (T3, N0, M0); IIIB: (T4, N0, M0). T3: T umor with direct invasion into any of the following: lung, brachiocephalic vein, superior vena cava, phrenic nerve, chest wall, or extrapericardial pulmonary artery or veins. T 4: T umor with invasion into any of the following: aorta (ascending, arch, or descending), arch vessels, intrapericardial pulmonary artery, myocardium, trachea, esophagus. N0: No regional lymph node metastasis. M0: No distant metastasis. (AJCC 8th ed.) 